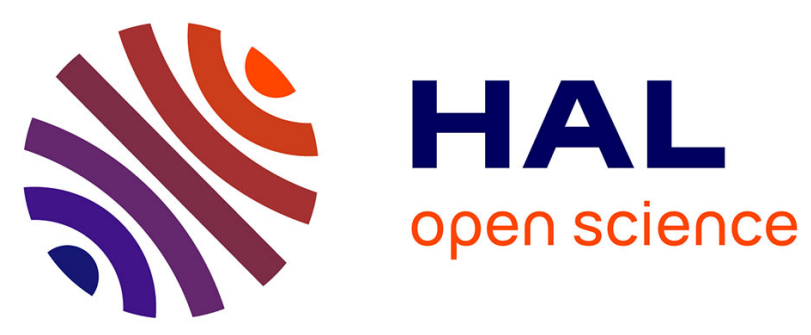

\title{
Executive functioning and risk-taking behavior in Parkinson's disease patients with impulse control disorders
}

Fanny Pineau, Emmanuel Roze, Lucette Lacomblez, Anne-Marie Bonnet, Marie Vidailhet, Virginie Czernecki, Jean-Christophe Corvol

\section{To cite this version:}

Fanny Pineau, Emmanuel Roze, Lucette Lacomblez, Anne-Marie Bonnet, Marie Vidailhet, et al.. Executive functioning and risk-taking behavior in Parkinson's disease patients with impulse control disorders. Journal of Neural Transmission, 2016, 123 (6), pp.573-581. 10.1007/s00702-016-1549-y . hal-01311564

\section{HAL Id: hal-01311564 \\ https: / hal.sorbonne-universite.fr/hal-01311564}

Submitted on 4 May 2016

HAL is a multi-disciplinary open access archive for the deposit and dissemination of scientific research documents, whether they are published or not. The documents may come from teaching and research institutions in France or abroad, or from public or private research centers.
L'archive ouverte pluridisciplinaire HAL, est destinée au dépôt et à la diffusion de documents scientifiques de niveau recherche, publiés ou non, émanant des établissements d'enseignement et de recherche français ou étrangers, des laboratoires publics ou privés. 
4 Fanny Pineau $^{\mathrm{a}, \mathrm{d}^{\star}} M A$, Emmanuel $\operatorname{Roze}^{\mathrm{a}} M D, P h D$, Lucette Lacomblez ${ }^{\mathrm{b}} M D, P h D$, Anne-Marie

5 Bonnet $^{\mathrm{a}} M D$, Marie Vidailhet ${ }^{\mathrm{a}} M D, P h D$, Virginie Czernecki ${ }^{\mathrm{c}} P h D$, Jean-Christophe Corvol $^{\mathrm{a}}$ $6 \mathrm{MD}, \boldsymbol{P h D}$.

$8{ }^{a}$ Sorbonne Universités, UPMC Univ Paris 06, and INSERM UMRS_1127 and CIC_1422, and

9 CNRS UMR_7225, and AP-HP, and ICM, Hôpital Pitié-Salpêtrière, Département des maladies du

10 système nerveux, F-75013, Paris, France

11 b Sorbonne Universités, UPMC Univ Paris 06, and INSERM UMRS_1146 and CIC_1422, and AP-

12 HP, and ICM, Hôpital Pitié-Salpêtrière, Département des maladies du système nerveux, F-75013,

13 Paris, France

$14{ }^{\mathbf{c}}$ Sorbonne Universités, UPMC Univ Paris 06, INSERM UMRS_975, and AP-HP, and ICM,

15 Hôpital Pitié-Salpêtrière, Département des maladies du système nerveux, F-75013, Paris, France

$16^{\text {d }}$ Service de Neurologie, Hôpitaux Civils de Colmar, 39 avenue de la liberté, F-68000, Colmar,

17 France

19 * Corresponding author:

20 Fanny PINEAU

21 fanny.pineau@ch-colmar.fr

22 Hôpitaux Civils de Colmar

23 Service de Neurologie

24 Bâtiment 59

2539 avenue de la Liberté

2668000 COLMAR

$27+33672055058$

30 Keywords: Parkinson's disease; Impulse control disorders; Executive functions; Risk-taking 31 behavior; Metacognitive skills

33 Acknowledgments: The authors thank the patients who participated to the study. 
37 Funding source of the study: none

39 Financial Disclosures/Conflict of Interest: FP has received grants from Novartis Pharma. EFR has 40 received grants from “poste d'accueil” APHP/CNRS, grants from INSERM (COSSEC), grants from

41 APHP (DRC-PHRC), grants from Fondation pour la Recherche sur le Cerveau (FRC), grants, 42 personal fees and non-financial support from Merz-pharma, grants and personal fees from Orkyn, 43 grants from IP Santé, grants and personal fees from Ultragenyx, personal fees from Novartis, 44 personal fees and non-financial support from Ipsen Pharma, non financial supports from Teva, non45 financial support from Abbvie, non-financial support from Dystonia Europe, non-financial support 46 from the Georgian Medical and Public Health Association, non financial support from the 47 International Federation of Clinical neurophysiology, non-financial support from the Movement 48 Disorders Society. LL has received personal fees from Novartis Pharma, personal fees from GE, 49 non-financial supports from Merck-Serono, grants from Sanofi-Aventis, grants from GSK, grants 50 from Cytokinetic. MV has received personal fees from Mertz, non-financial support from 51 Movement Disorders Society, non-financial support from EAN. JCC has received personal fees and 52 non-financial support from abbvie, personal fees from Amarentus, personal fees from Zambon, 53 personal fees from Pfizer, personal fees from Clevexel, non-financial support from UCB, grants 54 from Sanofi-Aventis, grants from Ipsen, grants from the Michael J. Fox foundation, and own stock 55 in B\&A Therapeutics. 


\section{ABSTRACT}

60 Background: Impulse control disorders (ICD) are common in Parkinson's disease (PD) and are

61 associated with dopaminergic medication. The purpose of this study was to investigate executive

62 function and risk-taking behavior in PD patients with ICD.

63 Methods: 17 PD patients with ICD (ICD-PD) were compared to 20 PD patients without ICD

64 (CTRL-PD) using neuropsychological and experimental tasks. Executive functions were assessed

65 using standard executive testing (Conner's Performance Test, Modified Wisconsin Card Sorting

66 Test, Trail Making Test and phonological verbal fluency). Subjects were also submitted to an

67 experimental gambling task consisted of three decks of money cards: neutral deck (equal

68 opportunity for gains as losses), winning deck (small amount of money with a positive balance) and

69 loser deck (high amount of money with a negative balance), evaluating risk-taking behavior

70 (number of cards picked in each deck) and valuation of the reward (subjective appreciation of the

71 value of each deck).

72 Results: There was no significant difference in executive functioning between groups. Both groups

73 selected more cards in the losing deck (high amount of money) as compared to the neutral deck

74 (Mann-Whitney test, ICD-PD, p=0,02; CTRL-PD, p=0,003) and to the winning deck (Mann-

75 Whitney test, ICD-PD $\mathrm{p}=0,0001$; CTRL-PD $\mathrm{p}=0,003)$, suggesting an increased risk-taking behavior.

76 Interestingly, we found that ICD-PD patients estimated the value of decks differently from CTRL-

77 PD patients, taking into account mainly the positive reinforced value of the decks (Mann Whitney 78 test, $\mathrm{p}=0,04)$.

79 Discussion: This study showed that executive pattern and risk-taking behavior are similar between

80 ICD-PD and CTRL-PD patients. However, ICD-PD patients showed a specific deficit of the

81 subjective estimation of the reward. Links between this deficit and metacognitive skills are 82 discussed. 


\section{INTRODUCTION}

Impulse control disorders (ICD) are behavioral disorders characterized by the failure to resist an impulse, inability to cut down and unsuccessful attempts to control a specific behavior (Evans et al. 2009). In Parkinson's disease (PD), the lifetime prevalence of ICD is about $14 \%$ (Weintraub et al. 2010). The most frequent ICD in PD are pathological gambling (PG), hypersexuality (HS), compulsive shopping (CS) and compulsive eating (CE) (Evans et al. 2009; Weintraub et al. 2010). The high prevalence of ICD in PD has been associated to the dopaminergic treatment, particularly to dopamine agonists (DA) (Weintraub et al. 2010). A possible hypothesis for the association between DA treatment and ICD involves their relative selectivity of D2-D3 dopamine-receptor. Those receptors are particularly abundant in the ventral striatum known to play a role in behavioral addiction and substance use disorders (Gurevich et al. 1999). In addition to DA exposure, clinical risk factors associated with ICD in PD are male gender, younger age, younger age at onset of PD and longer disease duration, personal or family history of alcohol or psychiatric disorders, high novelty seeking personalities, impulsivity and alexithymia traits (Weintraub et al. 2010; Voon et al. 2011a; Goerlich-Dobre et al. 2014). Although the pathological mechanisms remain largely unknown, the level of dopamine denervation of the fronto-striatal circuitry, involved in executive as well as decision-making functions, has been associated to ICD in PD (Cilia et al. 2011; Vriend et al. 2014). Especially, Cilia et al. (2011) found decreased prefrontal cortex, cingulate, insula, parahippocampal gyrus and striatal resting perfusion with increasing gambling severity in PG patients. These regions are involved in reward and risk processing, error detection, learning, decision-making and impulse control. Furthermore, the authors showed an anterior cingulate cortex-striatum disconnection, which could underline a specific impairment in ability to shift behavior after negative outcomes, leading patients to continue their behavior despite dramatic consequences.

A growing amount of studies attempted to investigate the cognitive characteristics associated with ICD in PD. Some studies found preserved cognitive functions (Siri et al. 2010; Djamshidian et al. 2011a; Mack et al. 2013). By contrast, a significant association between executive dysfunction and ICD has been demonstrated in few studies (Djamshidian et al. 2010; Vitale et al. 2011; Poletti et al. 2012). Especially, PD patients with PG had more severe impairments in retrieval of verbal and visuo-spatial information and cognitive flexibility (Santangelo et al. 2009). However, there were several limitations in these studies including materials used to explore cognitive functioning, often limited to working memory or global executive assessment. optimal selection in terms of rewarding or punishing outcomes between several alternative course 
119 of actions (Paulus 2005), has been well documented in PD patients (Delazer et al. 2009) and has 120 been specifically involved in PD patients with ICD (Djamshidian et al. 2010; Rao et al. 2010; Rossi 121 et al. 2010; Steeves et al. 2009). Especially, Rossi et al. (2010) found that PD patients with PG 122 obtained poorer performances in a risk-taking under ambiguity task that PD without PG. Using 123 delay-discounting tasks, several studies showed that altered impulsivity in PD with ICD can 124 contribute to risk-taking (Voon et al. 2010a; Housden et al. 2010). The physiopathology underlying 125 risk-taking behavior in PD with ICD has been explored and involved dysfunction in the reward 126 system including ventral striatum (Rao et al. 2010; Steeves et al. 2009). For example, using 127 functional magnetic resonance imaging to quantify resting cerebral blood flow (CBF) and blood 128 oxygenation level dependent (BOLD), Rao et al. (2010) showed that compared with non-ICD PD 129 patients, PD patients with ICD demonstrated significantly reduced resting CBF in the right ventral 130 striatum and significantly diminished BOLD activity in the right striatum during risk-taking. The 131 influence of pharmacological status on risk taking and impulsivity in PD with ICD has also been 132 explored (Voon et al. 2010b; Housden et al. 2010; Djamshidian et al. 2011a; Leroi et al. 2013). 133 These studies broadly concluded that PD patients with ICD tend to make more impulsive and risky 134 choices while ON dopamine agonist relative to those OFF dopamine agonists or without ICD. Self-awareness, metacognitive skills and their links to ICD have also been recently explored 136 (Brevers et al. 2013; Mack et al. 2013; Brevers et al. 2014). Self-awareness is usually evaluated by 137 questionnaire as the Beck Cognitive Insight Scale (BCIS), assessing the understanding of patients' 138 perspective about their anomalous experiences, their attribution and their aberrant interpretation of 139 specific life events. Impaired self-awareness or insight has been recognized as a feature of a large 140 number of neuropsychiatric disorders, including PD (Gilleen et al. 2010). Using the BCIS, Mack et 141 al. (2013) compared self-awareness of cognitive and behavioral issue in PD patients with and 142 without ICD and showed that PD patients with ICD are aware of their PD-related problems 143 including impulsivity. In a different perspective, Brevers et al. (2013; 2014) studied how 144 metacognitive sensitivity may influence gamblers without PD's decision-making. Metacognition 145 was assessed by asking participants to wager on their own decision. They found that gamblers tend 146 to wager high while performing poorly on the Iowa Gambling Task and in a non gambling task, 147 suggesting that pathological gamblers exhibit impaired metacognition in both gambling like and 148 more 'neutral' situations of decision-making.

The aim of the present study was to investigate executive functions with classical tasks and 150 risk-taking behavior using a task developed to assess behavioral response and valuation of the 151 reward in PD patients with ICD (ICD-PD) compared to PD controls patients without ICD (CTRL152 PD). 


\section{Subjects}

Patients were selected from among those attending the movement disorders unit of the Pitié-

157 Salpêtrière Hospital (Paris, France). All patients met the following inclusion criteria: idiopathic PD 158 according to the United Kingdom Parkinson's Disease Society Brain Bank and absence of dementia, 159 according to the MDS task-force criteria (Dubois et al. 2007). All patients obtained score higher 160 than 130 on the MDRS. Exclusion criteria were a history of ICD prior to PD-onset and treatment by 161 deep brain stimulation (DBS) before the ICD onset. Between January 2007 and January 2008, all 162 patients suspected to have ICD in the interview with the neurologist received a specific evaluation 163 of their ICD by a neuropsychologist. Presence and severity of active ICD were assessed with a 164 semi-structured interview assessing behavior and mood in PD, the 'Ardouin Scale of Behavior in 165 Parkinson's Disease' (ASBPD) (Ardouin et al. 2009; Rieu et al. 2015). Inclusion criteria for ICD166 PD patients was a score $\geq 2$ (moderate to severe) in at least one item in the ASBPD scale of 167 pathological gambling, compulsive eating, compulsive shopping and hypersexuality. The ASBPD 168 scale also evaluates compulsive DA and others hyperdopaminergic symptoms such as punding or 169 any form of hobbyism. Patients who presented punding or form of hobbyism without ICD were not 170 included in the study. CTRL-PD group was constituted of PD patients, matched with ICD-PD 171 patients for age, sex, educational level, disease's severity and duration, and who were candidate for 172 Deep Brain Stimulation (DBS) between the same period, without history of ICD. The CTRL-PD 173 patients were, therefore, at risks for ICD; their absence was confirmed by a score $\leq 1$ (none or mild)

174 in all ICD's items of the ASBPD scale, described below. Informed consent was obtained from all 175 individual participants included in the study.

\section{Procedure}

The two groups of patients underwent a comprehensive assessment of clinical, 179 neuropsychiatric and neuropsychological functioning. Assessment was performed in a single 180 session that lasted approximately 3 hours and when patients were in the 'on' state. Breaks were 181 introduced to avoid fatigue.

\section{Neurological assessment}

Patients underwent a neurological examination consisting of the motor section of the

185 Unified Parkinson's disease Rating Scale (UPDRS, section III) to measure the severity of motor symptoms in the 'on' state. Most of patients in both groups were at the motor fluctuations stage of

187 the disease. The demographic data (age, educational level), neurological details (age at PD onset 188 and PD duration) and treatments (medication type, total L-dopa equivalent daily dose (LEDD) and 
189 total L-dopa equivalent daily dose (LEDD) of dopamine agonists) of each patient enrolled were 190 recorded.

\section{Psychological assessment}

All patients underwent a psychological assessment consisting of the following:

194 1. the Montgomery and Asberg Depression Scale (MADRS) to evaluate depression, using only

195 the dysphoria factor defined by Suzuki et al. 2005, naming items of reported sadness, pessimistic

196 thoughts and suicidal thoughts to avoid confounding symptoms related to PD as 'lassitude', 'inability

197 to feel' or 'concentration difficulties;

198 2. the Starkstein scale (Starkstein et al. 1992) to identify apathy state and the severity of 199 apathetic symptom;

200 3. the Barrat Impulsiveness Scale (BIS-11) (Fossati et al. 2001), a global self-report scale of 201 impulsivity;

202 4. the 'Ardouin Scale of Behavior in Parkinson's Disease' (ASBPD) (Ardouin et al. 2009; Rieu 203 et al. 2015). The ASBPD consist of 21 items, grouped into four parts: general psychological 204 evaluation (part I), apathy (part II), non-motor fluctuations (part III) and hyperdopaminergic 205 behavior (part IV). Part I successively evaluates depressive mood, hypomanic mood, anxiety, 206 irritability and aggressiveness, hyperemotionality, and psychotic symptoms. Part II evaluates apathy 207 in behavioral terms, that is, activity level, cognitive level, and emotional level. Part III evaluates the 208 psychological state associated with the motor symptoms in the OFF and ON states in fluctuating 209 patients. Part IV assesses the presence and the intensity of behavioral disorders induced by 210 dopaminergic treatment, including nocturnal hyperactivity, diurnal somnolence, eating behavior, 211 creativity, hobbyism, punding, risk-taking behavior, compulsive shopping, pathological gambling, 212 hypersexuality, dopaminergic addiction, and excess in motivation. The timeframe of the assessment 213 is the preceding month. Each item is rated on a five-point scale (severe disorder, 4; marked disorder, 214 3; moderate disorder, 2; mild disorder, 1; absence of disorder, 0), by taking into account the severity 215 and the frequency of the disorder and its impact. The interview is completed by a psychiatrist, a 216 neuropsychologist, or a clinical psychologist familiar with PD and neuropsychiatric disorders in 217 movement disorders. Total completion time is approximately 1 hour.

\section{Neuropsychological assessment}

All PD patients underwent neuropsychological tasks to assess executive functioning and 221 risk-taking behavior. The Conner's Performance Test (CPT-II) (Connors 2004), a 15 minutes 222 computerized test, was used to evaluate attention and inhibition. The subject had to press the space 223 bar of the computer as soon as he sees any letter on the screen, except the letter $\mathrm{X}$, that he has to 
224 hold back and press nothing. Variability in reaction time (expressed in millisecond) was used to 225 assess attention capacity. Percentage of commission error (press when the letter is $\mathrm{X}$ ) referred to 226 inhibition abilities. The executive functioning was also assessed using : 1) conceptualization 227 capacities measured by the reached number of criterion (range from 0 to 6) in the Modified 228 Wisconsin Card Sorting Test (MCST) (Milner 1963); 2) shifting and reactive flexibility evaluated 229 by the difference between the time scores of TMT-B and TMT-A in the Trail Making Test (TMT) 230 (Reitan et al. 1985); 3) spontaneous flexibility and cognitive auto-activation skills using the 231 phonological verbal fluency $\mathrm{R}$ with the total number of correct words given in 2 minutes (Cardebat 232 et al. 1990).

233 To assess risk-taking behavior, we used a gambling task adapted from the Iowa Gambling 234 Task (IGT) (Bechara et al. 1994). The subject saw on a screen 3 decks of cards labeled A, B, and C. 235 Every time the subject picked a card, a message was displayed on the screen indicating the amount 236 of money he immediately won or lost. At the same time, on the top of the screen, the total amount 237 of money was displayed. The subject was asked to choose 50 cards and to win as much money as 238 possible. Contrary to the IGT, subject was notified that some decks were more advantageous than 239 others in order to avoid ambiguity and facilitate the comprehension of the rule. At the end of the 240 task, subject was asked to appreciate if each deck was a winning, a losing or a neutral deck (called 241 subjective variables). Subject can evaluate several decks as winning, losing or neutral. Decks had 242 been constructed so that deck A was a neutral deck (equal opportunity for gains as losses). Deck B 243 (small amount of money with a positive balance) was the winning deck with small gains but smaller 244 losses. Deck C can be considered as the losing deck (high amount of money with a negative 245 balance) as the subject won big gains but lost even more. Objective variables were the number of 246 cards chosen in each deck. The score for each objective variable ranges from 0 to 50 . The score for 247 each subjective variable (valuation of the reward by appreciation of each deck) ranged from 0 to 2 248 (0: loser deck; 1: neutral deck; 2: winner deck). This adaptation of the IGT was proposed to avoid 249 ambiguity and to focus on the ability to resist to a big risky reward for the benefit of a smaller but 250 safer reward rather than the capacity to detect and understand the rule. Especially, risk-taking 251 behavior is evaluated by comparing the number of cards picked in each deck, subjects being aware 252 of the advantageous/disadvantageous characteristic of the decks and constantly informed of his 253 immediate reward and the total amount of money. Moreover, due to subjective variables, this task takes into account the valuation of the reward.

\section{Statistical Analysis}

For demographic characteristics and neuropsychological data a Mann-Whitney U-test or a

258 Fisher exact test were used to compare CTRL-PD and ICD-PD groups. For risk-taking task, 
259 comparison between the 3 groups (HV, CTRL-PD, ICD-PD) and between the three decks (A, B, and

260 C) were performed by using a Kruskall Wallis test, followed when significant by a comparison of

261 each group by a Mann-Whitney U-test. All results were considered significant if the p-value was

262 less than 0.05 with no correction for multiple comparisons. Data were expressed as median +/-

263 upper and lower quartiles. Statistical analysis was performed using Statistica 9.1 software (StatSoft

264 France, F-94700, Maisons-Alfort).

\section{RESULTS}

\section{Patients' characteristics}

Thirty-seven patients (age range 33-69 years, men/women $=27 / 10$ ) participated in this 269 study. Seventeen patients were diagnosed as having one or more active ICD as the time of assessment. In ICD-PD group, specific criteria of the ASBPD confirmed presence of PG in 6 patients, HS in 1 patient, CS in 2 patients, CE in 2 patients and 6 patients had multiple ICD (i.e.

272 hypersexuality and pathological gambling or compulsive shopping and pathological gambling).

273 Twenty patients without history of ICD were included in the CTRL-PD group (score $\leq 1$ in all 274 ICD's items of the ASBPD scale).

The two groups did not differ in gender, age, educational level, age at PD onset, PD duration, LEDD dopamine agonist dose, UPDRS-III while "on" state and MDRS score (see results in table 1). However, the 2 groups differed in total LEDD ( $p=0,003)$, possibly because CTL-PD patients were recruited among those candidate for DBS and therefore needed more dopaminergic treatment to control the disease.

For neuropsychiatric characteristics, the two groups differed in MADRS dysphoria score $(\mathrm{p}$ $281=0,01)$, in Starkstein score $(p=0,01)$ and in the BIS-11 score $(p=0,003)$.

[Insert Table 1]

\section{Executive functions}

The two groups did not differ in attention capacities, conceptualization abilities, reactive

287 flexibility, spontaneous flexibility and inhibition capacities.

289 [Insert Table 2]

\section{Risk-taking behavior results}

For the risk-taking task, the two groups of PD patients were compared to fifteen healthy

293 volunteers (HV) matched in age, sex and educational level on the objective variables (number of 
cards chosen in each deck) and the subjective variables (appreciation of each deck: winning, neutral or losing deck).

For each selection, all patients were able to clearly identify the feedback they received.

First, we analyzed the pattern of performances inside each group. For HV, there was no significant difference between decks for both objective and subjective variables (Kruskall-Wallis test, $\mathrm{p}=0.61$ and $\mathrm{p}=0.13$ respectively). On the contrary, ICD-PD patients and CTRL-PD patients both showed significant differences of the number of cards in each deck (Kruskall Wallis, $\mathrm{p}=0.004$ for CTRL-PD, $\mathrm{p}=0.007$ for ICD-PD). For both groups, the number of cards chosen in the losing deck C (high amount of money) was higher as compared to the neutral deck A (Mann-Whitney test, ICD-PD, p=0,02 ; CTRL-PD, p=0,003) and to the winning deck B (small amount of money) (MannWhitney test, ICD-PD p=0,0001 ; CTRL-PD p=0,003). In addition, in the ICD-PD group, the number of card chosen in winning deck B was significantly lower than in neutral deck A (MannWhitney test, $\mathrm{p}=0.04)$. Subjective variables were not significantly different in the HV or the CTRL-PD groups (Kruskall-Wallis test, $\mathrm{p}=0.13$ and $\mathrm{p}=0.45$ respectively) whereas they were significantly different in the ICD-PD group (Kruskall-Wallis test, $\mathrm{p}=0.01$ ). ICD-PD patients evaluated the wining deck B (small amount of money) loser as compared to neutral deck A (MannWhitney, $\mathrm{p}=0.04$ ) and losing deck C (Mann-Whitney, $\mathrm{p}=0,01$ ).

Then, we compared the performances between the three different groups. When comparing PD patients to HV, we found no significant difference between HV and CTRL-PD groups for both objective and subjective variables. Furthermore, the ICD-PD group was significantly different from the HV for both variables: ICD-PD patients chose significantly less frequently the winning deck B (Mann Whitney, $\mathrm{p}=0,04$ ) and evaluated this deck more frequently as a losing one (Mann Whitney, $\mathrm{p}=0,02$ ) as compared to $\mathrm{HV}$. Moreover, ICD-PD patients evaluated the losing deck C more frequently as a winner than HV (Mann Whitney, p=0,02). ICD-PD patients and CTRL-PD patients did not significantly differ for any deck for objective variables. Furthermore, for subjective variables, ICD-PD patients evaluated the winning deck B more frequently as a loser one than the CTRL-PD patients (Mann Whitney test, $\mathrm{p}=0,04$ ).

\section{[Insert Table 3]}

\section{DISCUSSION}

This study examined executive functioning and risk-taking in PD patients with ICD. As previously observed by Weintraub et al. (2010) and Voon et al. (2011a), we found that ICD-PD patients showed greater impulsivity, more depressive elements and lack of motivation than PDCTRL patients. 
Concerning the executive functioning, we found that ICD-PD patients performed similarly

330 than CTL-PD patients, as supported by other studies, which showed no executive dysfunction

331 including set shifting, inhibitory process and reactive flexibility in ICD patients compared to CTRL

332 PD patients (Siri et al. 2010; Djamshidian et al. 2011a; Mack et al. 2013). This is against previous

333 reports showing a positive association between ICD and cognitive dysfunction (Santangelo et al.

334 2009; Vitale et al. 2011). Methodological and PD population's differences as well as small size of

335 groups can highlight those discrepancies.

336 In this study, we addressed to the patients an experimental task to investigate risk-taking

337 behavior and valuation of the reward. Our results showed that contrary to HV, both groups of PD

338 patients behave similarly, choosing more frequently cards in the loser deck (high amount of money)

339 compared to the other decks. These results confirm risk-taking behavior in PD with or without ICD

340 (Delazer et al. 2009; Djamshidian et al. 2010; Rossi et al. 2010). Risk-taking behavior in PD

341 probably involved dopamine replacement therapy's influence on mesolimbic spared circuit. For

342 example, Steeves et al. (2009) in a PET neuroimaging study in PD patients with PG demonstrated

343 decreased ventral striatal D2/D3 binding potential at baseline and a relatively greater decrease in

344 binding potential in the ventral striatum during performance of a gambling task. Consistent with our

345 results, Rao et al. (2010) found that both ICD-PD and CTL-PD groups behave similarly in a risk-

346 taking task. Interestingly, in that functional magnetic resonance imaging, the authors showed that

347 contrary to CTL-PD patients, ICD-PD patients demonstrated relatively diminished activity in the

348 ventral striatum during risk-taking.

349 Our results suggested also that ICD-PD patients showed a specific deficit of the subjective

350 estimation of the reward compared to patients without ICD, taking into account mainly the positive

351 reinforced value of the decks, and less considering the value of the loss. These results are in line

352 with studies exploring reinforcement sensitivity, demonstrating that via action on ventral striatal

353 dopamine function, dopamine replacement therapy could potentially alter reward responsiveness

354 and abilities to learn from negative decision outcomes (Franck et al. 2004; Pessiglione et al. 2006;

355 Piray et al. 2014). For example, Franck et al. (2004) showed that PD patients without ICD have

356 different learning and reward-seeking behaviors from healthy controls. PD patients showed exactly

357 opposite learning patterns during their medication ON and OFF states: PD patients achieved more

358 efficient learning by positive reinforcement during their ON medication state, whereas they

359 performed better through negative feedback during their medication OFF state. In ICD-PD patients,

360 it seems that dopamine agonist enhance the deviated learning pattern. Voon et al. (2010b) showed

361 that dopamine agonist enhance the rate of a gain-specific learning and increase striatal prediction

362 error activity observed in patient with ICD. Thus, ICD-PD patients can experiment a persistent

363 "better than expected" outcome while taking dopamine agonist. Dopamine agonists also enhance 
364 risk-taking behavior in ICD-PD patients (Voon et al. 2011b). While taking dopamine agonists, these 365 patients seem to have a bias towards risky choice independent of the effect of loss aversion. 366 Especially, Voon et al. (2011b) showed that neural activity in brain areas associated with risk 367 representation, such as the ventral striatum, orbitofrontal cortex and anterior cingulate cortex, are decreased in these patients.

In our study, both PD groups presented risk-taking behavior during the task but only ICD-

370 PD patients presented deficit in subjective appreciation of the reward. Especially, despite risk-taking 371 behavior, when they were asked about their perception of deck's value at the end of the task, CTL372 PD patients were able to correctly appreciate the reward. In contrary, ICD-PD patients presented a 373 specific deficit of the subjective estimation of the value of stimuli. Recent studies focusing on 374 influence of metacognitive skills on risk-taking process can probably contribute to understand this 375 specific deficit presented by our ICD-PD patients. Metacognition is the ability to cognizant and 376 have insight about the quality of the decision and to accurately judge whether the decision is surely 377 a good one or not. Brevers et al. (2013) studied how metacognitive sensitivity may influence gamblers without PD's decision-making during the Iowa Gambling Task. Metacognition was assessed by asking participants to wager on their own decision. They found that gamblers tend to wager high while performing poorly on the Iowa Gambling Task and that the difference was not due to reward/loss sensitivity, current clinical or cognitive status. The same authors in a more recent study (Brevers et al. 2014) replicated these results with a non gambling task (grammatical paradigm). They found that compared to healthy volunteers, pathological gamblers without PD also erroneously think that they are performing much better than they actually are. Both studies suggested that pathological gamblers exhibit impaired metacognition in both gambling like and more 'neutral' situations of decision-making. In our study, the erroneous appreciation of deck's values specifically presented by ICD-PD patients could be linked to deficit in metacognitive skills. Indeed, in these patients, introspection's abilities are possibly based on the under-optimal behavior and lead to focus on the positive reinforcement value. In that perspective, Djamishidian et al.

390 (2011b) showed that PD patients with ICD learn little of their mistakes, compared to ICD patients 391 without PD. On the contrary, a recent study evaluating self-awareness in PD patients with ICD 392 showed that presence of ICD was associated with awareness of impulsive behaviors, as indexed by 393 greater cognitive insight into thoughts and behaviors on the BCIS (Mack et al. 2013). In the 394 different studies cited above, different levels of awareness are probably involved and might explain 395 the discrepancies of the results. All together, these results showed that ICD-PD patients are 396 probably aware of ICD, but exhibit a fundamental impairment in their perception of winning or 397 losing behaviors among various situation of decision-making and provide an interesting perspective 398 to explain how metacognitive skills can contribute to the deficit of subjective estimation of the 
399

425 Ethical approval: All procedures performed in studies involving human participants were in 426 accordance with the ethical standards of the institutional and/or national research committee and 427 with the 1964 Helsinki declaration and its later amendments or comparable ethical standards. 


\section{REFERENCES}

Ardouin C, Chéreau I, Llorca PM, et al (2009) [Assessment of hyper- and hypodopaminergic behaviors in Parkinson's disease]. Rev Neurol 165:845-856. doi: 10,1016/j.neurol.2009.06.003 Bechara A, Damasio AR, Damasio H, Anderson SW (1994) Insensitivity to future

consequences following damages to human prefrontal cortex. Cognition 50:7-15.

$$
\text { Brevers D, Cleeremans A, Bechara A, et al (2013) Impaired self-awareness in pathological }
$$
gamblers. J Gamb 29:119-129. doi: 10.1007/s10899-012-9292-2

$$
\text { Brevers D, Cleermans A, Bechara A, et al (2014) Impaired metacognitive capacities in }
$$

individual with problem gambling. J Gamb Stud 30:141-152. doi: 10.1007/s10899-012-9348-3.

$$
\text { Cardebat D, Doyon B, Puel M, Goulet P, Joannette Y. (1990) Formal and semantic lexical }
$$

evocation in normal subjects. Performance and dynamics of production as a function of sex, age and educational level. Acta Neurol Belg 90:207-217.

$$
\text { Cilia R, Cho SS, Van Eimeren T, et al (2011) Pathological gambling in patients with }
$$

Parkinson's disease is associated with fronto-striatal disconnection: a path modeling analysis. Mov Disord 26:225-233. doi: 10.1002/mds.23480.

\section{Connors K. Conners's Continous Performance Test II (CPT-II V.5). 2004 Toronto: Multi-} Heath Systems Inc.

$$
\text { Delazer M, Sinz H, Zamarian L, et al (2009) Decision-making under risk and under ambiguity }
$$

in Parkinson's disease. Neuropsychologia 47:1901-1908. doi:

10.1016/j.neuropsychologia.2009.02.034.

$$
\text { Djamshidian A, Jha A, O'Sullivan SS, et al (2010) Risk and learning in impulsive and }
$$
nonimpulsive patients with Parkinson's disease. Mov Disord 25:2003-2010. doi:

\section{$10.1002 / \mathrm{mds} .23247$.}

$$
\text { Djamshidian A, O'Sullivan SS, Lees A, Averbeck BB (2011a) Stroop test performance in }
$$

$$
\text { impulsive and non impulsive patients with Parkinson's disease. Parkinsonism Relat Disord 17:212- }
$$



4602324.

214. doi: 10.1016/j.parkreldis.2010.12.014.

Djamshidian A, O'Sullivan SS, Wittmann BC, Lees AJ, Averbeck BB (2011b) Novelty seeking behavior in Parkinson's disease. Neuropsychologia 49:2483-2488. doi:

10.1016/j.neuropsychologia.2011.04.026.

$$
\text { Dubois B, Burn D, Goetz C et al (2007) Diagnostic procedures for Parkinson's disease }
$$

dementia: recommendations from the movement disorder society task force. Mov Disord. 22:2314-

Evans AH, Strafella AP, Weintraub D, Stacy M (2009) Impulsive and compulsive behaviors in PD. Mov Disord 24:1561-1570. doi: 10.1002/mds.22505.

Fossati A, Di Ceglie A, Acquarini E, Barratt ES (2001) Psychometric properties of an Italian version of the Barrat Impulsiveness Scale-11 (BIS-11) in nonclinical subjects. J Clin Psychol $57: 815-828$

Franck MJ, Seeberger LC, O'Reilly RC (2004) By carrot or by stick : cognitive reinforcement learning in parkinsonism. Science 306:1940-1943.

Gilleen J, Greenwood K, David A (2010) Lack of Insight and Awareness in Schizophrenia and Neuropsychiatric Disorders, in Neuropsychiatric Disorders, $1^{\text {st }}$ Edition, Edited by Miyoshi K, Morimura Y, Maeda K. Springer Publising. 33-49

Goerlich-Dobre KS, Probst C, Winter L, et al (2014) Alexithymia-An independent risk factor for impulsive-compulsive disorders in Parkinson's disease. Mov Disord 29:214-220. doi: $10.1002 / \mathrm{mds} .25679$

$$
\text { Gurevich EV, Joyce JN (1999) Distribution of dopamine D3 receptor expressing neurons in }
$$
the human forebrain: comparison with D2 receptor expressing neurons. Neuropsychopharmacology 20:60-80.

Housden CR, O'Sullivan SS, Joyce EM, Lees AJ, Roiser J (2010) Intact reward learning but

8 elevated delay discounting in Parkinson's disease patients with impulsive-compulsive spectrum 9 behaviors. Neuropsychopharmacology 235:2155-2164. doi: 10.1038/npp.2010.84. 
Dopaminergic influences on executive function and impulsive behavior in impulse control disorders

482 in Parkinson's disease. J Neuropsychol 7:306-325. doi: 10.1111/jnp.12026.

Mack J, Okai D, Brown RG, et al (2013) The role of self-awareness and cognitive dysfunction in Parkinson's disease with and without impulse-control disorder. J Neuropsychiatry Clin Neurosci 25:141-149. doi: 10.1176/appi.neuropsych.12030076.

Milner B (1963) Effects of different brain lesions on card sorting: the role of the frontal lobes. Arch Neurol 9:90-100.

$$
\text { Paulus MP (2005) Neurobiology of decision-making: quo vadis? Brain Res Cogn Brain Res }
$$
23:2-10.

$$
\text { Pessiglione M, Seymour B, Flandin G, Dolan RJ, Frith CD (2006) Dopamine-dependent }
$$
prediction errors underpin reward-seeking behavior in humans. Nature 442:1042-1045.

$$
\text { Piray P, Zeighami Y, Barhami F, Eissa AM, Hewedi DH, Moustafa AA (2014) Impulse control }
$$
disorders in Parkinson's disease are associated with dysfunction in stimulus valuation but not action valuation. J Neurosci 34:7814-7824. doi: 10.1523/JNEUROSCI.4063-13.2014.

$$
\text { Poletti M, Bonuccelli U (2012) Impulse control disorders in Parkinson's disease: the role of }
$$
personality and cognitive status. J Neurol 259:2269-2277. doi: 10.1007/s00415-012-6506-6.

$$
\text { Rao H, Mamikonyan E, Detre JA, Siderowf AD, Stern MB, Potenza MN, Weintraub D (2010) }
$$

Decreased ventral striatal activity with impulse contrl disorders in Parkinson's disease. Mov Disord 25:1660-1669. doi: 10.1002/mds.23147.

$$
\text { Reitan RM, Wolfson D. The Trail making Test. } 1985 \text { Tucson, A.Z: Neuropsychological Press. }
$$

$$
\text { Rieu I, Martinez-Martin P, Pereira B, et al (2015) International validation of a behavioral scale }
$$

in Parkinson's disease without dementia. Mov Disord 30:705-713. doi: 10.1002/mds.26223.

$$
\text { Rossi M, Gerschcovich ER, De Achaval D, et al (2010) Decision making in Parkinson's }
$$
disease patients with and without PG. Eur J Neurol 17:97-102. doi: 10.1111/j.1468- 
and pathological gambling in patients with Parkinson's disease. Mov Disord 24:899-905. doi:

508 $10.1002 / \mathrm{mds} .22472$.

Siri C, Cilia R, De Gaspari D, et al (2010) Cognitive status of patients with Parkinson's disease and pathological gambling. J Neurol 257:247-252.doi: 10.1007/s00415-009-5301-5. Starkstein SE, Mayberg HS, Leiguarda R, Preziosi TJ, Robinson R (1992). A prospective longitudinal study of depression, cognitive decline, and physical impairments in patients with Parkinson's disease. J Neurol Neurosurg Psychiatry 55:377-382.

Steeves TD, Miyasaki J, Zurowski M, et al (2009) Increased striatal dopamine release in Parkinsonian patients with pathological gambling: a [11C] raclopride PET study. Brain 132:13761385. doi:10.1093/brain/awp054.

Suzuki A, Aoshima T, Fukasawa T, et al (2005) A three-factor of the MADRS in major depressive disorder. Depress Anxiety 21:95-97.

Vitale C, Santangelo G, Trojano L, et al (2011) Comparative neuropsychological profile of pathological gambling, hypersexuality and compulsive eating in Parkinson's disease. Mov Disord 26:830-836. doi: 10.1002/mds.23567.

Voon V, Reynolds B, Brezing C, et al (2010a) Impulse choice and response in dopamine agonist-related impulse control behaviors. Psychopharmacology 207:645-659. doi: 10.1007/s00213$009-1697-y$.

Voon V, Pessiglione M, Brezing C, et al (2010b) Mechanisms underlying dopamine-mediated reward bias in compulsive behaviors. Neuron 65:135-142 doi: 10.1016/j.neuron.2009.12.027. Voon V, Sohr M, Lang AE, et al (2011a) Impulse control disorders in Parkinson's disease: a multicenter case-control study. Ann Neurol 69:986-996. doi: 10.1002/ana.22356.

Voon V, Gao J, Brezing C et al (2011b) Dopamine agonists and risk: impulse control disorders in Parkinson's disease. Brain 134:1438-1446. doi: 10.1093/brain/awr080. 
532 impulse control disorders in Parkinson's disease. Mov Disord 29:904-911. doi: 10.1002/mds.25886.

533 Weintraub D, Koester J, Potenza MN, et al (2010) Impulse control disorders in Parkinson's

534 disease: a cross-sectional study of 3090 patients. Arch Neurol 67:589-595. doi:

535 10.1001/archneurol.2010.65. 


\section{TABLES}

Table 1: Demographic and clinical aspects of PD patients with and without ICD

Values are median (lower-upper quartile). P-value: Mann-Whitney test between groups.

\begin{tabular}{l|c|c|c} 
& $\begin{array}{c}\text { ICD-PD } \\
\mathrm{n}=17\end{array}$ & $\begin{array}{c}\text { CTL-PD } \\
\mathrm{n}=20\end{array}$ & $\mathrm{p}$ \\
Characteristics & $14 / 3$ & $13 / 7$ & 0,24 \\
\hline Men/Women, No & $55(37-69)$ & $55(40-62)$ & 0,52 \\
Age (Yr) & $7(3-7)$ & $7(3-7)$ & 0,45 \\
Education (Yr) & $48(32-65)$ & $48(35-55)$ & 0,57 \\
Age at PD onset (Yr) & $7(2-10)$ & $5,5(4-12)$ & 0,60 \\
PD duration (Yr) & $897,5(299,88-$ & $1049,89(527,05-$ & 0,003 \\
& $1247,33)$ & $1549,84)$ & 0,78 \\
LEDD (mg/dose) & $299,94(77-718)$ & $340,23(66,68-700)$ & 0,62 \\
LEDD dopamine agonist (mg/dose) & $7(0-23)$ & $8,5(0-34)$ & 0,01 \\
UPDRS-III score while on state & $6(0-13)$ & $1,5(0-7)$ & 0,01 \\
Dysphoria specific MADRS score & $7(3-14)$ & $4(0-10)$ & 0,07 \\
Starkstein scale score & $140(133-144)$ & $139(131-143)$ &
\end{tabular}

Table 2: Neuropsychological compares between patients with PD with and without ICD.

Values are median (lower-upper quartile). P-value: Mann-Whitney test between groups.

\begin{tabular}{c|c|c|c} 
& $\begin{array}{c}\text { ICD-PD } \\
\mathrm{n}=17\end{array}$ & $\begin{array}{c}\text { CTL-PD } \\
\mathrm{n}=20\end{array}$ & $\mathrm{p}$ \\
\hline $\begin{array}{c}\text { Impulsivity } \\
\text { Global BIS-11 }\end{array}$ & $63(48-81)$ & $52(36-70)$ & 0,003 \\
Attention & $88,5(4,74-202,2)$ & $82,9(3,05-193,4)$ & 0,68 \\
RT variability CPT & & & \\
Executive functioning & & $6(3-6)$ & 0,23 \\
criterion number MSCT & $34(6-149)$ & $45,2(10-78)$ & 0,56 \\
TMT B-A & $26(6-43)$ & $20(8-38)$ & 0,08 \\
fluency R & $22,2(4,7-38,9)$ & $20,8(5,6-70,8)$ & 0,67 \\
\% commission CPT &
\end{tabular}


Table3: Compares between the two PD groups and the healthy volunteers on the risk-taking task

Values are median (lower-upper quartile). P-value: Kruskal-Wallis test for each deck between groups.

${ }^{a}$ Significantly different from $\mathrm{HV}$; ${ }^{b}$ Significantly different from $C T L-P D ;{ }^{c}$ Significantly different from deck B ;

${ }^{d}$ Significantly different from deck C, Mann Whitney test.

\begin{tabular}{c|c|c|c|c} 
& $\begin{array}{c}\text { ICD-PD } \\
\mathrm{n}=17\end{array}$ & $\begin{array}{c}\text { CTL-PD } \\
\mathrm{n}=20\end{array}$ & $\begin{array}{c}\text { HV } \\
\mathrm{n}=15\end{array}$ & $\mathrm{p}$ \\
\hline $\begin{array}{c}\text { Objective variables } \\
\text { Number of cards neutral deck A }\end{array}$ & $17(7-14)^{d}$ & $15(8-23)^{d}$ & $15(6-24)$ & 0,58 \\
Number of cards winning deck B & $14(5-18)^{a, d}$ & $15(5-26)^{d}$ & $17(10-33)$ & 0,01 \\
Number of cards losing deck C & $19(12-33)$ & $20(12-34)$ & $16(10-30)$ & 0,21 \\
Subjective variables & & & & \\
Appreciation neutral deck A & $2(0-2)^{c}$ & $1(0-2)$ & $1(0-2)$ & 0,24 \\
Appreciation winning deck B & $0(0-2)^{a, b, d}$ & $1,5(0-2)$ & $2(0-2)$ & 0,04 \\
Appreciation losing deck C & $2(0-2)^{a}$ & $1,5(0-2)$ & $0(0-2)$ & 0,06
\end{tabular}

